\title{
Modeling and Simulation in Numerical Computation Education
}

\author{
Lian Xue $\mathrm{e}^{1, \mathrm{a}^{*}}$ \\ ${ }^{1}$ School of Computer and Computing Science, Zhejiang University City College \\ Hangzhou, 310015, P.R. China \\ $a^{*} x u e l @ z u c c . e d u . c n$
}

Keywords: Scientific computing; Modeling; Design; Dynamic simulation

\begin{abstract}
In this paper, we make some discussion on the concept of teaching reform in the "numerical computation" of education through modeling and simulation, which enable students further master the background, idea and mechanism of the numerical algorithm embedded in "numerical computation" education as well as conducive to improving their autonomous learning ability and exploring spirit.
\end{abstract}

\section{Introduction}

When it comes to computer education, "numerical computation" becomes a popular topic owing to that it is widely used for resolving issues among financial ,economic and other fields by means of simulation algorithm on the basis of computer engineering. Generally speaking, it often presents a imitate relationship with computer development [1][2]. As the upgrading of computer hardware and software instruments has become more and more frequently, it is imperative for the traditional "numerical computation" education to transform its concept or mode to a new one in order to keep in pace with times.

For most of the time, modeling and simulation is often considered as an approach for transforming realistic matters into mathematical ones. And the basic mathematical concepts and means as well as computer tools are indispensable for resolving these models [3][4]. Recently, modeling and simulation has received extensive attention among the teaching reform of computer education on account of the growing popularity of computer engineering and its application.

Among the education of "numerical computation", modeling and simulation deserves extensive attention because we can take advantage of the methods of modeling and simulation for better cultivating and enhancing exploration ability and creative spirit [5][6]. In our paper, we put forward several significant advices for the reform of "numerical computation" teaching by importing the concept of modeling and simulation.

The remainders of this article are divided into four segments. In section 2, we introduce the modeling and simulation so as to promote students' comprehension on the background of algorithm. In the following section, this paper emphasizes on how to shape the fundamental philosophy of algorithm among modeling and simulation. Then the application of GUI in computer is discussed in detail in the next section. At last, we summarize this paper and make some conclusions.

\section{Using modeling and simulation to help students understand the background of algorithm}

Nowadays, education about "numerical computation" is often deemed as deriving from realistic matters, which involves various numerical computation methods. When applying these methods, a basic principle is to comply with rigorous logic of the questions to be studied and conduct a study gradually under the guidance of mathematical deduction, meaning of geometric, computational formula and some other directions need to be taken into consideration. 
As for students, "numerical computation" is nothing more than calculation formula which is denoted by various notations of mathematical and process of argumentation for convergence. Thus the application of "numerical computation" is simply making full use of different mathematical methods and some related theory. In this case, the origin and background about "numerical computation" is often neglected among the process of teaching. In fact, the background of which tends to be understood during the process of utilizing the algorithm of modeling and simulation to resolve realistic matters, simulating, analysis of data, meanwhile, it is conducive to stimulating the enthusiasm for math study as well as the improvement of algorithm.

For example: if we run a program in the context of MATLAB (i.e. a kind of widely used computer software)

figure

set (gcf,'menubar','none')

axes ('position', [llllll)

$[x, y]=$ ginput

And then you should put your hand on the pop-up window, clicking the right position to match with required interpolation points, after that you can press enter in order to obtain the entire coordinates parameters of the interpolation points. Why the simulation of your hand is able to realize simply rely on certain coordinates parameters after you taking back your hand? In reality, the grasp some knowledge about interpolation method enables you take advantage of the so called "parameter curve" to interpret the matter. Parameter curve often involve the selection of interpolation points among parameter intervals, and the utilization of spline interpolation function is applied to fitting the approximate value at $\mathrm{m}$ points, then a graphic composed of $\mathrm{m}$-point is presented.

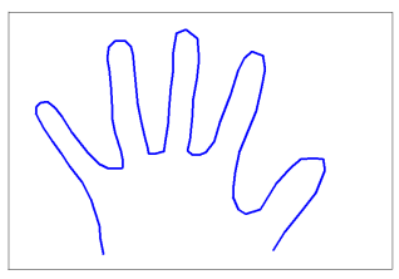

Figure 1. Drawing up your hand on the screen based on some coordinates

Through the case of researching the shape of the hand above, you are able to discover more data by applying numerical means as well as analyzing a bit of data to confirm whether the numerical methods applied meet the requirement of the context of the problem. And the thought of modeling and simulation thought is in sustainable utilization, indicating that modeling and simulation is unquestionable becoming a powerful carrier to probe the ability of students to develop.

In the emergence and development of modeling and simulation, mathematical foundation on intuitive, interoperability, repeatability, exploratory are indispensible embedded. When confronted with realistic matters, the effectively use of modeling and simulation methods will help students grasp such skills or abilities as intuitive observation, hands-on operation, independent analysis, repeated probe. Therefore, in the practice of "numerical calculation" teaching, modeling and simulation ought to be paid extensive attention it deserves, and then the students' inquiry ability can be improved continuously.

\section{Using modeling and simulation to help students form basic concepts of algorithms}

During the process of tackling the mathematical matters, the traditional ways tend to be the directional thinking mode that following the procedures from condition to conclusion. While for some of the ways of this mode to resolve the problem, students still not able to think broadly or propose solutions. 
Probing matters with the help of modeling and simulation methods will benefit us by overcoming or circumventing the obstacles to the matter, getting over the thinking difficulties, and thus to form a novel way of thinking or creative ways to tackle the matter.

In the education of "numerical computation", there is a great deal of concepts or knowledge embedded in some algorithms origin from such fields as engineering, economic, and so on. With the help of the mathematical trial, students could have access to grasp the basic skill simply by abstracting the idea and procedure of the numerical algorithm that rooted in the real world, and the conclusions could be perorated and formed into a significant entirety. In the meanwhile, students will gradually comprehend the algorithm as well as build a novel concept or accumulate new knowledge.

For example, the truncation error among "numerical calculation" provide us the possibility for obtaining the numerical calculation error [7] [8], but it is not an unique way. It is obvious that student may have difficulty in grasping this. Nevertheless, if you use extremely small $h$ to conduct the experiment, especially in the process of calculating high-order derivatives, we will better understand the numerical results of the error will not decrease when $h$ reach a certain extent instead reach a larger level ( As shown in Figure 2)! In fact, when the value of step h is kept in a low level, the result of the error appears larger due to the existence of rounding error. By making full use of these vivid visual trials, the students are provided an opportunity to further probe the concept included in "truncation error" as well as the relationship with the rounding error, and also promote the ability of students to take the motivation to probe the matter simultaneously

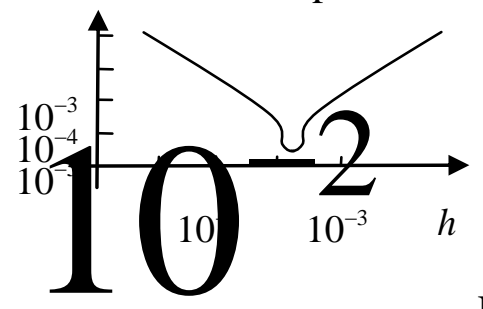

Figure 2. Picture of relation of and error

As shown in the figure above, in the reform of teaching resort to modeling and simulation is equivalent to direct students to apply model on the basis of computer, to improve their programming skill as well as an approach to transform mathematical learning from a tedious theoretical study into a significant procedure indispensible for investigation.

\section{Using modeling and simulation to help students analyze algorithm deeply}

The software MATLAB is often referred to and widely used during the process of the construction of "numerical computation" education. As we know, the formidable computation ability presented by the software MATLAB makes it possible for us to fulfill our unique tasks such as drawing a vector graph freely and conveniently. Thus the application of this software will become more and more promising. As far as I know, the software of MATLAB has owned a dominant position among such fields as scientific as well as engineering computing.

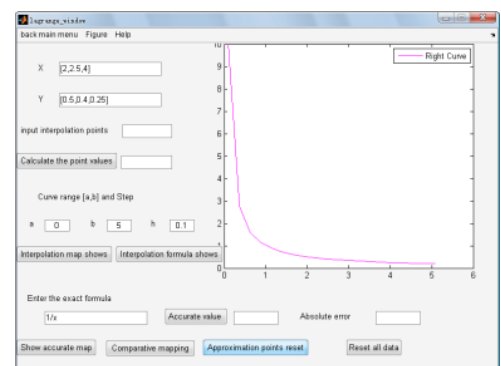

Figure 3. Lagrange Interpolation

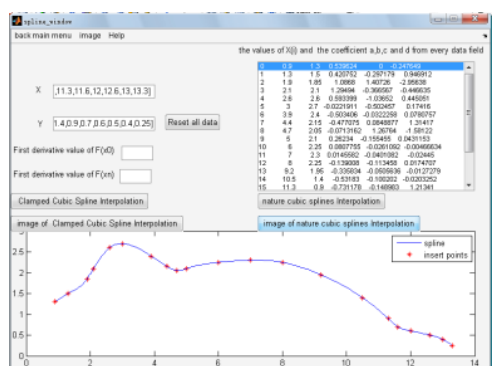

Figure 4. Spline Interpolation

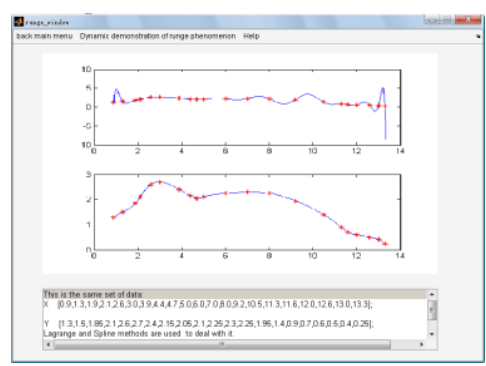

Figure 5. Runge Phenomenon 
For the purpose of better understanding the function of this algorithm by figures and results, we are able to resort to GUI contact surface design which is rooted in the software of MATLAB [9][10], which is conducive to further understanding the principle of "numerical computation". And we can obtain the designated parameters, diagrams and error analysis with clicking controls in a form, through which the experimenters are capable of grasp the related algorithms from the perspective of computer implementation.

Taking interpolation as an example, the entire types of the advantages and disadvantages interpolation can be divided into four (Namely Lagrangian interpolation, Newton interpolation, piecewise linear interpolation and cubic interpolation). As presented in figure 3 and figure4, they represent the Lagrangian interpolation and cubic spline interpolation, respectively. With the help of the two figures, it is not difficult for us to analyze their nature and error. As shown in Figure 5, the famous "Longge phenomenon" tell us why the Lagrangian interpolation is not steady and it will appear at both ends of the interval to vibrate. Thus students will have an in-depth understanding about Lagrange interpolation.

\section{Conclusions}

In summary, students will have an opportunity to find out the laws with not all-inclusive summarization in some unique cases by applying the modeling and simulation, and they are required to put forward their hypothesis and testify them step by step. So it is the combination of preparation process, observation analysis as well as summarized guess. Thus we can conclude that modeling and simulation is not simply a significant "thought experiment" but a valid approach to cultivate the ability required for probe as well.

\section{References}

[1] Z.C.Shi, The Third Scientific Method- Scientific Computation in the Computing time, Tsinghua University Press, Beijing, 2000.

[2] Z.Li, Application of Math Experiment in the University Mathematics Teaching, Neijiang Technology, 28(2007)11-14.

[3] Q.Fu, A Brief Talk on the Students General Ability of Cultivating, Higher Engineering Education Research, 12(2002)86-88.

[4] D.Kincaid, Numerical Analysis :Mathematics of Scientific Computing, Tsinghua University Press, Beijing, 2003.

[5] J.Liu, Application of GUI in Matlab for the Information and Computing Science Specialty Teaching, Wuhan Technology, 20(2007)12-14.

[6] R.L.Burden, J.D.Faires, Numerical Analysis, Higher Education Press, BeiJing, 2001.

[7] C.N.Yang, On the application of math teaching and research, Yang Zhenning Speech Set, Nankai University Press, TianJin, 1985.

[8] Q.Fu, On the Students General cultivate the ability of students, Higher Engineering Education Research, 12(2002)86-88.

[9] Z.Li, Experimental Math at the University Mathematics Teaching, Neijiang Technology, 28(2007)11-12.

[10] Q.Fu, Brief discussion about cultivating the students' comprehensive ability, Researches in Higher Education of Engineering, 12(2002)86-88. 